\title{
Research and Implementation of Key Technology for Calculation of Acquiring Simulation Training System
}

\author{
Xuedong Xue ${ }^{1}$, Shuai Zhang $^{1}$, Ya Luo ${ }^{1}$, Jian Qin $^{1}$, Qinglan Zhao ${ }^{2}$ \\ ${ }^{1}$ Ordnance NCO Academy,Army Engineering University, Wuhan Hubei, China \\ ${ }^{2}$ Military Representative Office of the 207 Institute, Taiyuan Shanxi, China
}

\begin{abstract}
For a missile acquiring system, there are problems such as high system failure rate, poor acquiring computer maintainability and loopholes in the calculation program during the training process of the troops. For this purpose, the acquiring professional training can get rid of the dependence on the actual equipment, and provide good hardware conditions for the training of the troops and the teaching of the college, a missile acquiring simulation training system is designed and constructed. The key to acquiring simulation training system software design is acquiring calculation, and the two technical difficulties in acquiring calculation are data format conversion and data output precision control. This paper focuses on the basic algorithms and implementation methods of self-test calculation, data format conversion and data output precision control, and solves the key technical problems of acquiring calculation in the acquiring training software.
\end{abstract}

\section{Introduction}

With the continuous development of computer technology, simulation technology and electronic technology, the simulation training technology has also developed rapidly, especially in the fields of civil aviation, military equipment, industry, etc., and it is widely used. A type of missile acquiring training has always relied on the actual installation. For equipment loss, there are problems such as high system failure rate, poor computer maintainability and loopholes in the calculation program, and are affected by the site and weather factors. On the basis, a missile acquiring simulation training system is designed and developed

The acquiring calculation data is input according to a certain format, in which $\mathrm{A} 1, \mathrm{~A} 2, \mathrm{~A} 3$, and $\mathrm{A} 4$ are input in the format of "degree, minute, and second", and A5, A6, $\mathrm{A} 7, \mathrm{~B} 0, \mathrm{C} 1$, and $\mathrm{C} 2$ are input in the format of "degree, minute". When converting the format of "degree, minute, second" or "degree, minute" into the format of "degree", extracting "degree", "minute", "second" from the input data must be extracted according to the specified format, but it becomes a difficult point that extract "minutes" and "seconds".

The data output results of "angle calculation" and "self-test calculation" are expressed in the format of "degree, minute", so the format of the "degree" of the output data must be converted into the format of "degree, minute". The precision control of "minutes" is 2 digits after the decimal point, rounding off from the third decimal place. The calculated result may be expressed in the form of scientific notation, so it is more difficult to round off the calculation. The solution is to first convert the representation of the scientific notation into a representation of the non-scientific notation, and then round off the operation.

When some special cases in the rounding operation are not considered in detail, the output will produce some deviations. The solution is to carry out seriously and repeatly tests to find out all possible special cases, thus solving this difficult problem well. This paper focuses on self-test calculation and data output format conversion and precision control methods.

\section{System software design}

The system software program is cured in the general hand held computer, not only can achieve the aiming calculation function, but also modified design loopholes in the original aiming calculation program, designed the failure query module for the aiming equipment. The system software is divided into three modules: aiming at calculation, data management and printing. System software used Microsoft eMbedded Visual Basic and eMbedded Visual $\mathrm{C}++$ two tools for software development, software calculation module and data query module with eMbedded Visual Basic program to write, print function by the eMbedded Visual $\mathrm{C}++$ program. The software developed with eMbedded Visual Basic and eMbedded Visual $\mathrm{C}++$ can run on Windows $\mathrm{CE}$ operating system platforms [1-3]. System software in the aiming calculation module is mainly carried out angle calculation and self-test calculation, the system of software composition is shown in Fig. 1. 


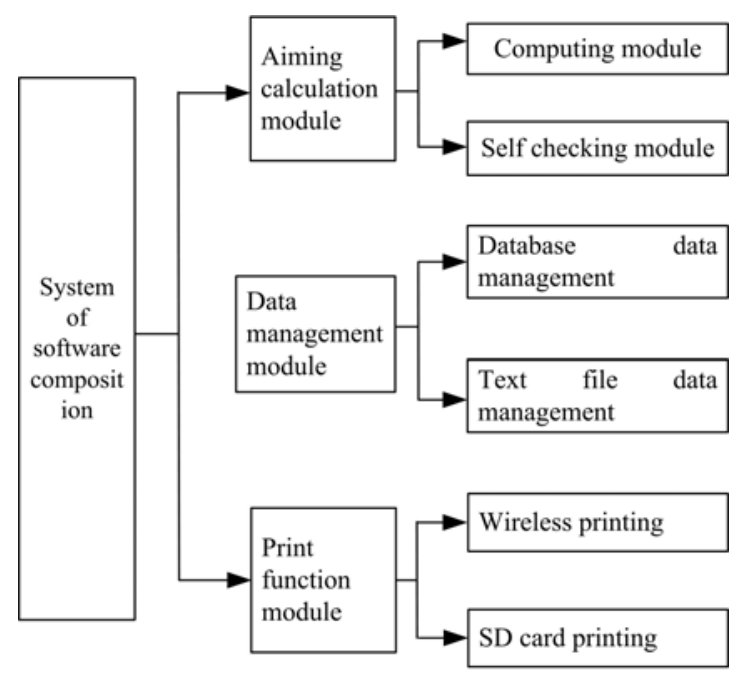

Fig. 1. The system of software composition.

\section{Self-test calculation method}

\subsection{Calculation formula}

$$
\begin{aligned}
& \mathrm{V}=\mathrm{A} 4-\mu \\
& \beta \mathrm{A}=\mathrm{V}-(\mathrm{C} 2-\mathrm{C} 1)=\mathrm{W} \\
& \beta \mathrm{B}=\mathrm{A} 7-\mathrm{B} 0 \\
& \mathrm{P}=\beta \mathrm{A}-\beta \mathrm{B}=\mathrm{W}-(\mathrm{A} 7-\mathrm{B} 0)
\end{aligned}
$$

\subsection{Basic algorithm}

First, convert A1, A2, A4, and B0 from "degree, minute, and second" format to "degree" format, and convert A7, $\mathrm{C} 1$, and C2 from "degree, minute" format to "degree" format; Check the validity of the data, then just check the two data of $\mathrm{A} 7$ and $\mathrm{C} 1$, so that they are between $-360^{\circ}$ - $+360^{\circ}$; Finally, perform self-test calculation, get the self-test value $P$, and judge the output result Acquiring at the correctness of the operation. The aiming calculation software self checking program is shown in Fig. 2.

\subsection{Implementation method}

$$
\begin{aligned}
& \text { tu }=\text { test_Degree }(\mathrm{a} 1+\mathrm{a} 2) \\
& \text { tv }=\text { test_Degree }(\mathrm{a} 4-\mathrm{tu}) \\
& \text { tba }=\text { test_Degree }(\mathrm{tv}-(\mathrm{c} 2-\mathrm{c} 1)) \\
& \text { tbb }=\text { test_Degree }(\mathrm{a} 7-\mathrm{b} 0) \\
& \mathrm{p}=\text { tba }- \text { tbb } \\
& \mathrm{p}=\text { test_Degree }(\mathrm{p}))^{\prime} \text { test_Degree }(\mathrm{p}) \text { function controls }
\end{aligned}
$$
the range of $P$ between 0 and 360

If $\mathrm{p}>180$ Then ', control the value range of $\mathrm{P}$ between -180 and 180 degrees

$$
\mathrm{p}=\mathrm{p}-360
$$

End If

If $p<0$ Then

$\mathrm{p}=\mathrm{p} *(-1)$

zjjg_Label = "-" + Trans_Degree(p)

Else

End If

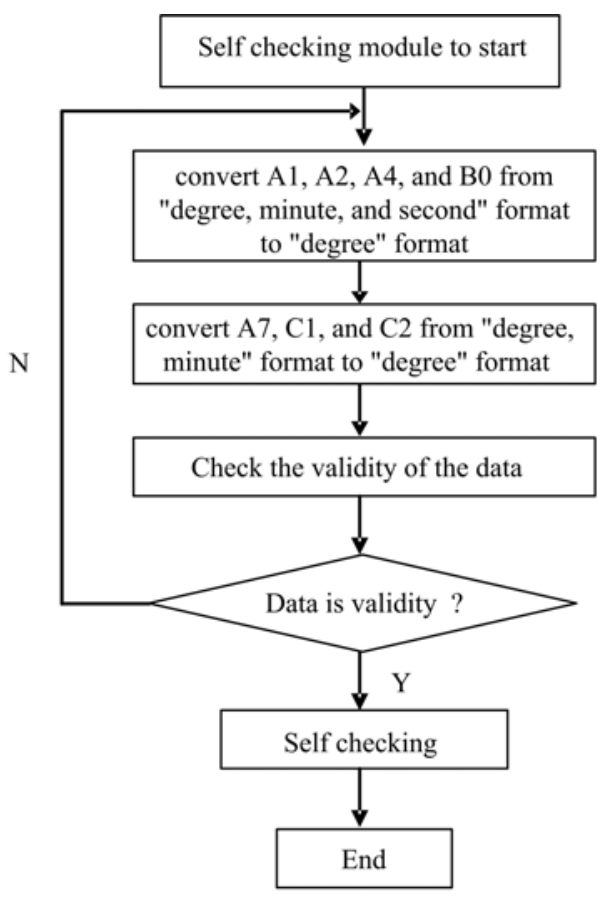

Fig. 2. The aiming calculation software self checking program.

\section{Data output format conversion and precision control method}

The data output results of "angle calculation" and "selftest calculation" are expressed in the format of "degree, minute", so the format of the "degree" of the output data must be converted into the format of "degree, minute", and "minutes" The precision needs to be controlled 2 digits after the decimal point and rounded off from the 3rd decimal point. The results calculated in this way may be expressed in the form of scientific notation. It is more difficult to round off the calculation. First, the representation of the scientific notation is converted into the representation of the non-scientific notation, and then rounded off. When some special cases in the rounding operation are not detailed, the output will produce some deviations. The solution is to carry out serious and repeated tests to find out all possible special cases, which is a good solution to this difficult problem[4-7].

\subsection{Basic algorithm}

The specific algorithm is, in the first step, if there is a scientific notation, the scientific notation needs to be converted into an unscientific notation[8-11]. First, the input data must be greater than 0.00008 , otherwise the value is 0 ; then the position of "E/e" is obtained and the index after "E/e", the index is generally negative, not less than -5 , otherwise the value is 0 ; then the integer part of the scientific notation is obtained, and the integer part of the scientific notation is multiplied according to the different conditions of the index. 0.1, 0.001, 0.0001, 0.00001 , which is converted into an unscientific counting method; the second step is to obtain the integer part of "degree" and the integer part of "minute"; the third step is to obtain the position of the decimal point and 
according to the position of the decimal point To get the value of 2 digits after the decimal point and the value of the 3rd digit after the decimal point, you need to consider the following special cases:

i. When the first two digits of the "minute" are less than 10, the first digit of the fractional part of the "minute" is added with 0 ;

ii. If the value of the third digit after the decimal point is not less than 5, round to the first two digits after the decimal point of the "minute";

iii. When the first two digits of the fraction of "minute" become 100, the integer part of the "minute" is carried and the first two digits of the fraction of "minute" are assigned 00 ;

iv. When the integer part of the "minute" is rounded to 60 , the integer part of the "degree" is carried over, and the integer part of the "minute" is assigned a value of zero.

\subsection{Algorithm programming}

The programming is as follows:

Dim str Temp, str1, str2 As String

$/ / \mathrm{dbl}$ in range: 0 360, Digit uncertainty

Dim int Pos, int E As Integer

// The case of scientific notation: when the total number of digits is greater than 16

Dim s_return, dbl Temp, dbl minute As

Double //Numbers greater than 1 and less than 15 digits are not represented by scientific notation

Dim intDu, int minute, int minute Xiao As Integer

Dim str minute Xiao As String

If $\mathrm{dbl}$ in $<0$ Or dbl in $>360$ Then

Msg Box "Trans_Degree ( ) Input parameters are outside the range of 0 to 360 !", vb Critical, "Acquiring calculation"

Trans_Degree $=$ "Input parameters are outside the range of $\overline{0}$ to $360 . "$

Exit Function

End If

If $(\mathrm{dbl}$ in $<0.000083333333333)$ Then

'0.00008 $\approx 0^{\circ} 0.005^{\prime}$, can be seen as 0

Trans_Degree $=" 0^{\circ} 0.00^{\prime \prime}$

Exit Function

End If

Str Temp $=$ CStr $(\mathrm{dbl}$ in $)$

Dbl Temp $=$ dbl_in

If (In Str (1, str Temp, "E", vb Text Compare) <> 0)

Or (InStr(1, strTemp, "e", vbTextCompare) $>>0)$ Then

If $(\operatorname{InStr}(1$, strTemp, "E", vbTextCompare $)=0)$ Then

$/ /$ Get the location of E/e

Int Pos $=\operatorname{InStr}(1$, strTemp, "e", vb Text Compare)

Else

Int Pos $=\operatorname{InStr}(1$, str Temp, "E", vb Text Compare)

End If

str1 = Mid(strTemp, intPos +1 , Len(strTemp) intPos) ' Index behind $\mathrm{E}$

$\operatorname{int} E=\operatorname{Int}(\operatorname{str} 1)$

str1 = Mid(strTemp, 1, 8) data.

// The index is negative and requires up to 8 digits of $\mathrm{dbl}$ Temp $=\mathrm{CD}$ bl $(\operatorname{str} 1)$

Select Case int E //index is negative, up to five digits, otherwise the assignment is 0

Case -1:

Case -2:

$$
\mathrm{dbl} \text { Temp }=\mathrm{dbl} \text { Temp } * 0.1
$$

Case -3:

$$
\mathrm{dbl} \text { Temp }=\mathrm{dbl} \mathrm{Temp} * 0.01
$$

Case -4:

$$
\mathrm{dbl} \mathrm{Temp}=\mathrm{dbl} \mathrm{Temp} * 0.001
$$

Case -5:

$$
\mathrm{dbl} \text { Temp }=\mathrm{dbl} \mathrm{Temp} * 0.0001
$$

End Select

End If

$$
\text { Int } \mathrm{Du}=\mathrm{Fix}(\mathrm{dbl} \text { Temp) } / /
$$

The integer part of the degree of acquisition

Dbl minute $=\mathrm{dbl} \mathrm{Temp}-$ int $\mathrm{Du} / /$ Get minute

Dbl minute $=\mathrm{dbl}$ minute $* 60$

Int minute $=$ Fix $(\mathrm{dbl}$ minute $) \quad / /$

Get the integer part of the

score

Str Temp $=$ CStr $(\mathrm{dbl}$ minute $)$

' Msg Box (strTemp)

Int Pos = In Str(1, str Temp, ".", vb Text Compare) str1 $=$ Mid(str Temp, int Pos $+1,2)$

// Take the value of 2 digits after the decimal point, such as 8.1234567 is 12 str2 $=\operatorname{Mid}($ str Temp, int Pos $+3,1)$

// Take the value of the third digit after the decimal point, such as 8.1234567 is 3

Int minute Xiao $=\operatorname{Int}(\operatorname{str} 1)$

If int minute Xiao $<10$ Then

// If the first two digits of the fractional decimal point are less than 10 , the " 0 " in "01" will be removed, and 0 needs to be added to the front.

Str minute Xiao = "0" + CStr(in minute Xiao)

Else

Str minute $\mathrm{Xiao}=\mathrm{CStr}($ int minute Xiao $)$

End If

If $\operatorname{Int}(\operatorname{str} 2)>=5$ Then

// If the value of the third digit after the decimal point is $\geqq 5$, round off to the two decimal places.

Int minute $\mathrm{Xiao}=$ int minute $\mathrm{Xiao}+1$

If int minute Xiao $<10$ Then

// If the fractional part of the minute is less than 10 , the " 0 " in " 01 " will be removed, and 0 needs to be added to the front.

Str minute Xiao $=" 0 "+C S t r($ int minute Xiao $)$

Else

Str minute $\mathrm{Xiao}=\mathrm{CStr}($ int minute Xiao $)$

End If

If int minute Xiao $=100$ Then

// When the first two digits of the fractional decimal point are incremented to 100 , the integer part of the fraction is rounded, and the first two digits of the fractional decimal value are assigned 00.

Int minute $=$ int minute +1

Int minute Xiao $=0$

Str minute Xiao = "00" 
If int minute $=60$ Then

// When the integer part of the fraction becomes 60 after the carry, the integer part of the degree is rounded, and the integer part of the fraction is assigned a value of 0 .

Int $\mathrm{Du}=$ int $\mathrm{Du}+1$

Int minute $=0$

End If 'If int minute $=60$

End If 'If int minute Xiao $=100$

End If 'If $\operatorname{Int}(\operatorname{str} 2)>=5$

Trans_Degree $=$ CStr(int Du $)+$ "o" + CStr(int minute $)$ + "." + str minute Xiao + "minute" // Get the answer

\subsection{Calculation aids for data verification}

Before inputting each parameter for calculation, the known data of the position is compared with the measured data to check whether the acquiring operation measurement data is incorrect. Its interpretation criteria are:

$|(\mathrm{C} 2-\mathrm{A} 5)-(\mathrm{A} 4-\mathrm{A} 3)|<0.0083333^{\circ}=0.5^{\prime}$

The meaning of the measurement data: $\mathrm{C} 2$ is to check the benchmark value, A5 is the main benchmark measurement value, $\mathrm{C} 2-\mathrm{A} 5$ is the angle between the main benchmark point and the acquiring point and the angle between the benchmark point and the acquiring point.

Known data meaning: A4 check the large local angle of the benchmark, A3 main standard benchmark large local angle, A4-A3 also indicates the angle between the main benchmark point and the acquiring point and check the line between the benchmark point and the acquiring point.

Therefore, in theory $|(\mathrm{C} 2-\mathrm{A} 5)-(\mathrm{A} 4-\mathrm{A} 3)|=0$, but in practice, due to the existence of measurement and operational errors, $|(\mathrm{C} 2-\mathrm{A} 5)-(\mathrm{A} 4-\mathrm{A} 3)| 0$, the allowable error range is less than 0.5 minutes. If the program checks that the value is greater than 0.5 , it indicates that the measurement or operation is incorrect.

The verification of the input data can avoid the misalignment of the acquiring accuracy due to the measurement or operation error, reduce the influence of human factors, and make the missile acquiring operation more accurate and reliable.

\section{Conclusion}

On the basis of fully considering the problems caused by the training of a certain missile aiming system and the actual operation, the simulation training system is constructed by means of hardware simulation missile, launcher and aiming device. The system is fully capable of simulating the full functionality of the training system in the installation, and the failure rate of the lighting system of the aiming equipment during the training process is high, aiming at poor computer maintenance and the existence of loosening of the calculation program.
Teaching problems have been improved, so that aimed at professional training to get rid of the dependence on the actual equipment to solve the missile launch vehicle subsystem training bottlenecks for the training of troops and institutions of teaching to provide good hardware conditions, thus Improve the training level of the troops and the teaching level of the institutions, and achieved remarkable military economic benefits.

\section{References}

1. LIANG Gang, WANG Chong-hua.SyStem modeling of port container crane training Simulator based on object-orientedmethod [J].CHINESE JOURNAL OF CONSTRUCTION MACHINERY, 2006-1, 4(1): 108-109.

2. ZHANG Guo-yu, et al.An Optoelectronic Inspection Method for the Taper of a Bore [J].ACTA ARMAMENTARII, 2007-2, 28(2): 108-109.

3. Fang Zejun, Experimental Study of the Impact of Fuel Additive on Combustion \& Emission Performance of Gasoline Engine [D], Beijing University of Technology, 2008.

4. Dai Jianhua \& Li Kaicheng, Measurement of High Current Based on Rogowski Coil [J], High Voltage Engineering,2002,28 (1) : 1-4.

5. Gu Yunhui, Liu Yabin, "Research on Fault Diagnosis Based on Automatic Testing System"[J]. Micro-Computer Information, 2005, 7-1, 146-148.

6. Mohamad Sawan, Faycal Mounaim, Guillaume Lesbros. Wireless monitoring of electrode-tissues interfaces for long term characterization $[\mathrm{J}]$.Analog Integrated Circuits and Signal Processing. 2008, 55(1).

7. Wei Baohua, Yang Suochang, Zheng Silong et al, Development Model of Open Circuit Testing System, J. Ordnance Industry Automation, 2004, 23(5): 76-7.

8. Zhang Xianda.Modern Signal Processing [M].published by Tsinghua University

9. Kopfu, Feserk. Rejection of Narrow-band Noise and Repetitive Pulses in On-site PD Measurements [J]. IEEE Transactions on Dielectrics and Electrical Insulation, 1995, 2(6): 1180-1191.

10. Xue Xuedong, Cheng Xude, Bai Weibing et al.TimeFrequency Analysis of PD Signals Based on Matlab and STFT [J]. Application Research of Computers, 2009, 6(2): 128-130.

11. Wang Guoli, Hao Yanpeng, Li Yanming. Analysis of local discharge signal based on adaptive spectrum [J]. Journal of Xi'an Jiao Tong University, 2001, 35 (8): 790-794. 